\title{
Cornell Spectrum Imager: Open Source Spectrum Analysis with ImageJ
}

\author{
Paul Cueva, ${ }^{*}$ Robert Hovden, ${ }^{*}$ and David A. Muller,*** \\ * School of Applied and Engineering Physics, Cornell University, Ithaca, NY 14853 \\ ** Kavli Institute at Cornell for Nanoscale Science, Ithaca, NY 14853
}

Here we present a free, open-source software tool for spectral analysis of electron energy loss (EELS), energy dispersive X-ray (EDX), or cathodoluminescence (CL) data - the Cornell Spectrum Imager (CSI) (Fig. 1) [1]. Spectral imaging, whether via EELS, EDX or CL, is a powerful approach to analyzing materials in a wide array of fields [2]. Proper analysis of spectral data requires software for extracting the chemical signatures present in a spectrum. Basic analysis requires background extrapolation, background subtraction, and signal integration [3]. However, the commercial software available for spectrum analysis remains an expensive, complicated, and time-consuming black box. Worse, licensing terms generally restrict the software to a single computer tied to the dataacquisition microscope or a handful of machines. For user facilities, educational institutes, or any other setting where multiple users on a single tool can be expected, the limited availability of software becomes the bottleneck to data analysis, user training, and throughput. Faced with several hundred users logging over 10,000 hours on our instruments at Cornell, we decided to write a universal data analysis tool that could be freely distributed, would run on all computers, and in order to minimize training, would present the same user interface for imaging, EELS and EDX data analysis. Our goal was software that could be run with minimal instruction or training - this meant ensuring all options needed for basic analysis were immediately visible to the user, and also limiting those options to avoid overwhelming a new user.

CSI aims to provide an efficient, intuitive user interface that also includes unique features. Built as a plugin for the ImageJ platform (Fig. 1), CSI offers an approachable graphical interface supported by standard image analysis software. Users on PC, Mac, or Linux can work with multi-gigabyte one, two, or three-dimensional data-sets. In its current realization, CSI can read DigitalMicrograph, Emispec, image stack, or datacube files containing a single spectrum, line profile, or map. Once the data has been read into ImageJ, the spectrum analyzer offers various unique capabilities: The option of point, line, rectangle, circle, and freehand region selection on spectrum images enables analysis of irregularly shaped features. Additional features currently include Gaussian fitting and principal component analysis (PCA) that allow for smoothing and higher-level analysis of fine structure variations [4]. CSI is optimized for generating single or composite chemical maps (Fig. 2, 3) from three-dimensional spectral data sets, either by integration (Fig. 2) or principal components (Fig. 3, 4) $[5]$.

\section{References}

[1] Released under Creative Commons Attribution-NonCommercial-ShareAlike 3.0 License

[2] R.F. Egerton, Rep. Prog. Phys., 72 (2009) 016502

[3] D.C. Joy and D.M. Maher, J. Microsc, 124 (1981) 37-48

[4] J. Verbeeck and S. Van Aert, Ultramicroscopy, 101 (2-4) (2004), 207-224.

[5] Funded by DOE BES Award \#DE-SCOO02334 and CNS (NSF \#EEC-0117770, 06465470). We thank Marion Stevens-Kalceff, Chee Chia, Stephen Joseph, Paul Munroe (UNSW), Julia Mundy and Huolin Xin (Cornell) for example data sets to test and demonstrate the software. 


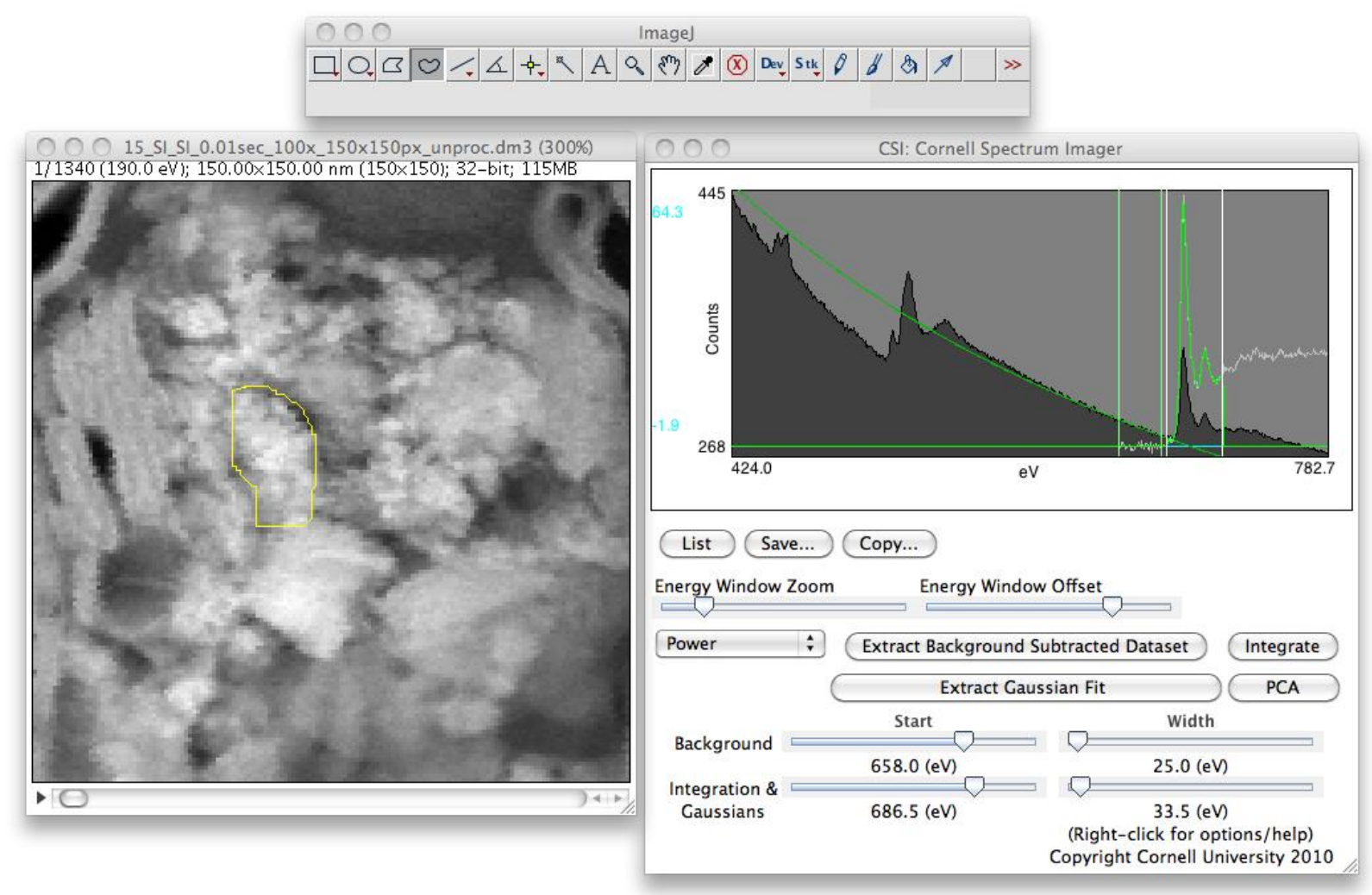

Fig. 1 - Screenshot of CSI performing analysis of a 3D EELS dataset. Averaged energy loss spectrum (right, dark gray) from selected freehand region of dataset (left, yellow) is shown with power-law background extrapolation and autoscaled background-subtracted spectrum. By setting appropriate background and integration windows (right, vertical lines), one can immediately obtain a chemical map from the integrated intensity of each spectrum contained at every pixel.

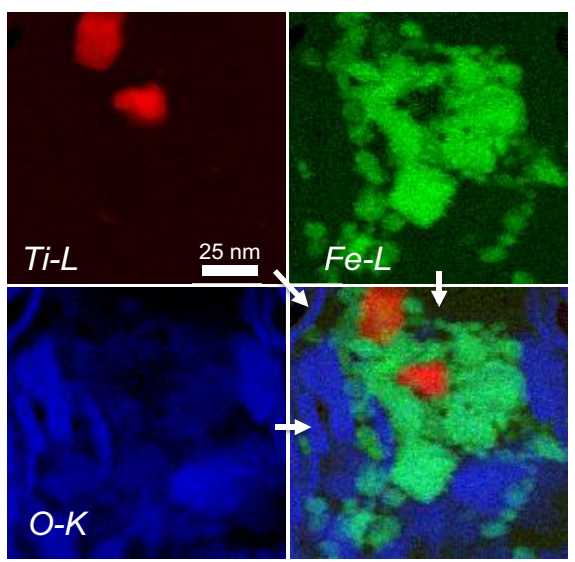

Fig. 2 - RGB compositional background-subtracted EELS map of a ferralsol soil (see Fig. 1) rendered by CSI and ImageJ. (Data from C. Chia, S. Joseph, P. Munroe, UNSW, and H. L. Xin, Cornell)

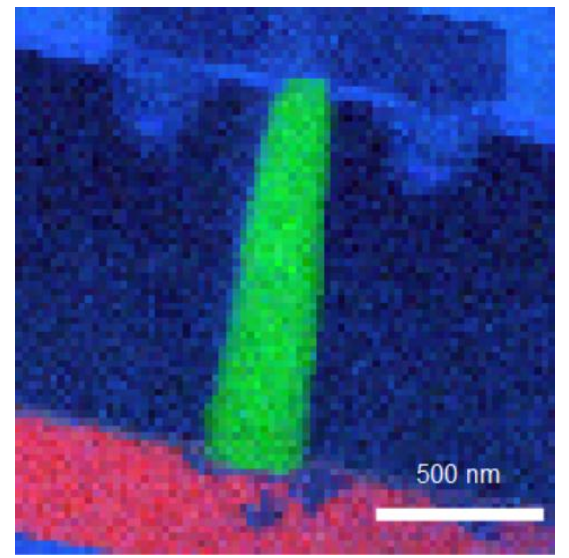

Fig. 3 - Mean-centered PCA of an X-ray map of a PPC750, showing the first 3 principle phases, that capture the copper interconnect $(\mathrm{R})$, the tungsten via $(G)$, and the silicon in the transistors (B).

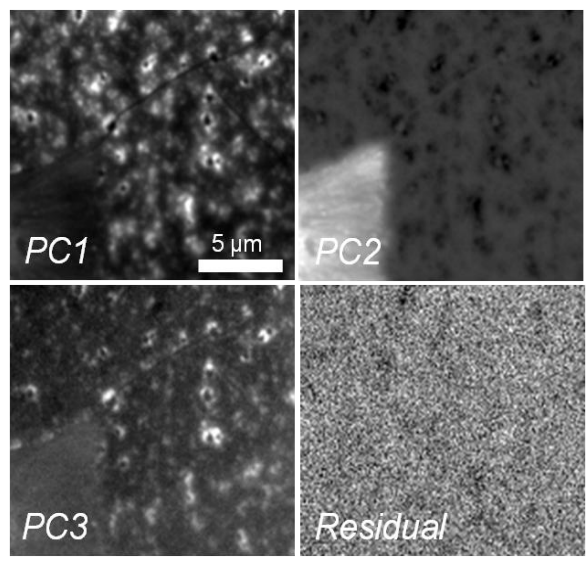

Fig. 4 - First three principal components and remaining residual from $\mathrm{CL}$ data of AlN/GaN obtained on a JEOL $7001 \mathrm{~F}$ equipped with a Gatan XiCLone cathodoluminescence system. (Data from M. Stevens-Kalceff, UNSW) 